\title{
Loi de commande prédictive pour le positionement des axes d'une machine outil à architecture ouverte
}

\author{
Mara Susanu ${ }^{1, a}$, Didier Dumur ${ }^{1}$, Christophe Tournier ${ }^{2}$ et Claire Lartigue Th $^{2,3}$ \\ 1 Supélec, Service Automatique, Plateau de Moulon, 3 rue Joliot-Curie, 91192 Gif-sur-Yvette Cedex, France \\ 2 LURPA-ENS de Cachan, 61 avenue Président Wilson, 94235 Cachan Cedex, France \\ ${ }^{3}$ IUT de Cachan Paris XI, 9 Ave. Division Leclerc, 94234 Cachan Cedex, France
}

Reçu le 14 février 2005, accepté le 13 avril 2005

\begin{abstract}
Résumé - Concevoir une CN pour machine-outil selon une structure à architecture ouverte permet d'en améliorer la flexibilité en autorisant l'intégration de modules spécifiques. Dans ce contexte, l'article envisage en premier lieu l'ajout d'un module de commande d'axe basé sur une stratégie prédictive avancée. Cette stratégie incluant une anticipation en boucle fermée s'avère particulièrement performante en termes de suivi de consigne et de facilité d'implantation. Considérant le centre d'usinage MIKRON, des tests réalisés en simulation avec cette structure de commande, et comparés aux essais effectués avec une commande classique, valident l'apport de ce module. Une deuxième étape examine ensuite les perspectives apportées par l'intégration d'un module avancé de génération de consignes pour trajectoires d'usinage surfacique.
\end{abstract}

Mots clés : Commande prédictive / trajectoire d'usinage / architecture ouverte / MOCN

Abstract - Predictive Control for axis positioning of machine-tool under an open-architecture framework. The CNC design of machine-tool under an open architecture framework improves flexibility by enabling integration of user-built specific modules. In this direction, this paper considers first addition of axis control modules based on advanced predictive control structures. Such strategy including a closed-loop feedforward action appears to be particularly efficient in terms of tracking performance and implementation ability. Considering as example the MIKRON machining centre, tests realized in simulation with this predictive control structure, compared to experiments achieved on the machine with the classical axis control architecture, validate the proposed approach. A second step examines perspectives provided by the integration of a specific module dedicated to advanced path generation for surface machining trajectories.

Key words: Predictive control / machining toolpath / open architecture / CNC

\section{Introduction}

Les performances demandées aux commandes numériques de machine-outil s'avèrent de plus en plus sévères, tant en terme d'augmentation de la cadence d'échantillonnage (Usinage Grande Vitesse), qu'en terme de précision de découpe par exemple (inférieure au micron désormais). La satisfaction des spécifications imposées implique d'une part l'utilisation d'actionneurs pour la commande d'axe de plus en plus performants, d'autre part l'implantation de lois de commande dites avancées, permettant une optimisation du comportement du système (stabilité, rapidité, précision et robustesse). Cependant, s'il s'avère assez facile de changer

\footnotetext{
a Auteur pour correspondance:

mara.susanu@supelec.fr
}

d'actionneur, la structure de commande actuellement implantée sur les commandes numériques est le plus souvent complètement fermée, y inclure une technique de commande avancée est alors impossible.

À partir de ce constat, il apparaît nécessaire de définir une structure dite «ouverte », au sein de laquelle l'utilisateur pourrait simplement implanter sa propre loi de commande par adjonction d'un module supplémentaire. Le maître mot de ces architectures ouvertes est dès lors modularité, imposant la présence de petits modules indépendants, reliés par des interfaces de communication. Un système ouvert doit être conçu de sorte que des applications correctement implantées puissent fonctionner sur un large éventail de plates-formes provenant de différents fournisseurs [1], qu'elles puissent communiquer avec d'autres applications, et que l'ensemble reste cohérent aux yeux de l'utilisateur. En conséquence, les 
recherches dans ce domaine visent à accroître les fonctionnalités du système, lui conférant une plus grande « portabilité » et un caractère « reconfigurable » très prononcé [2]. L'objectif ultime de ce type de structure est bien sûr de réduire les coûts et d'augmenter la flexibilité, en facilitant la « réutilisation » de logiciels par exemple, ou l'intégration de modules spécifiques utilisateur.

L'article présente, au travers d'une structure de commande numérique générique, dite RST, parfaitement adaptée au contexte d'architecture ouverte, et en s'appuyant sur l'exemple concret du centre d'usinage MIKRON cinq axes (UGV), l'apport d'un module de commande d'axes de machine-outil de type prédictif. Pour cela, et du fait de la configuration fermée des commandes actuelles, une machine-outil « virtuelle » a été mise au point. Elle reproduit de façon suffisamment précise le comportement du centre d'usinage pour permettre de comparer les performances obtenues avec la commande d'axes prédictive par rapport aux caractéristiques relevées avec des commandes d'axes classiques. L'article envisage également, toujours dans ce contexte d'architecture ouverte, l'apport supplémentaire en terme de performance en positionnement d'un module avancé de génération de trajectoire.

La structure de l'article est la suivante. La section 2 examine les phases de modélisation et d'identification nécessaires à l'élaboration de la machine « virtuelle », ainsi que la validation du comportement obtenu. La section suivante détaille tout d'abord les étapes théoriques requises pour l'élaboration du module de commande prédictive généralisée (GPC), depuis la mise sous forme RST jusqu'à la définition du critère de commande. Elle compare ensuite les résultats obtenus sur la machine « virtuelle» avec cette stratégie à ceux enregistrés sur site. La section 4 étudie enfin la mise en œuvre d'un module de génération de trajectoire apportant des perspectives plus larges que les modules actuels.

\section{2 Élaboration de la machine-outil « virtuelle »}

Afin de tester en simulation l'impact de stratégies avancées dédiées à la commande d'axe, et à défaut de pouvoir le faire sur la machine, il est nécessaire de concevoir un environnement virtuel reproduisant le plus précisément possible le comportement des modules de commande du centre d'usinage MIKRON UCP 710, la précision du modèle impliquant la pertinence des futurs résultats en simulation. Pour simplifier la démarche, et sans perte de généralité, seuls les axes $X, Y$ et $Z$ du centre d'usinage sont considérés par la suite.

\subsection{Structure générale}

Si l'on se restreint aux modules de génération de trajectoire et de commande d'axe de la commande numérique, l'ensemble peut se représenter par le schéma

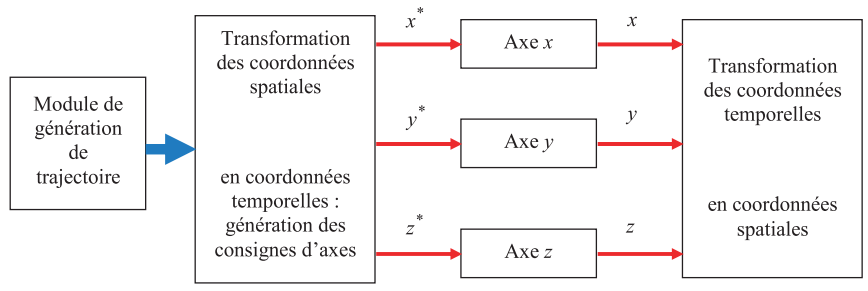

Fig. 1. Structure générale d'une commande numérique.

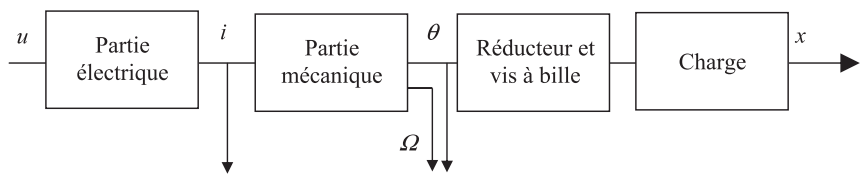

Fig. 2. Modélisation du servomoteur.

simplifié de la figure 1. Dans le but de valider la nouvelle structure de commande, seuls les modules reproduisant le comportement des axes sont décrits ci-dessous. Il est clair que la mise au point d'une machine-outil « virtuelle » nécessite également le développement du module de génération de trajectoire, ce qui n'est pas abordé ici. Dès lors, les trajectoires simulées appliquées en consigne de chacun des axes seront celles prélevées sur la machine lors des essais comparatifs.

Par ailleurs, l'environnement virtuel développé est conçu avec les hypothèses simplificatrices suivantes, conférant malgré tout un comportement très similaire au comportement réel. Tout d'abord, les axes sont supposés découplés (Fig. 1), sans interaction entre eux; ensuite au regard des dynamiques mises en jeu, les servomoteurs alternatifs sont modélisés par des moteurs à courant continu. Ces hypothèses ne permettent certes pas de reproduire certaines caractéristiques très fines du fonctionnement, mais sont largement suffisantes pour élaborer une comparaison réaliste des structures de commande.

\subsection{Modélisation}

Si l'on considère dans un premier temps l'ensemble constitué du servomoteur et de la charge (ceci pour chacun des axes envisagé), et selon les hypothèses énoncées précédemment, le schéma-bloc global de cette structure est donné figure 2. Les fonctions de transfert tout à fait classiques intervenant dans les différents blocs sont rappelées ci-dessous. Le lecteur pourra consulter [3] pour plus de détails sur l'obtention de ces relations.

Pour la partie électrique :

$$
\begin{array}{r}
H_{u i}(s)=\frac{f+J_{\mathrm{tot}} s}{\left(K_{\mathrm{c}} K_{\mathrm{e}}+R f\right)+\left(R J_{\mathrm{tot}}+L f\right) s+L J_{\mathrm{tot}} s^{2}} \\
(s \text { variable de Laplace })
\end{array}
$$

Pour la partie mécanique :

$$
H_{i \Omega}(s)=\frac{K_{\mathrm{c}}}{f+J_{\text {tot }} s}
$$




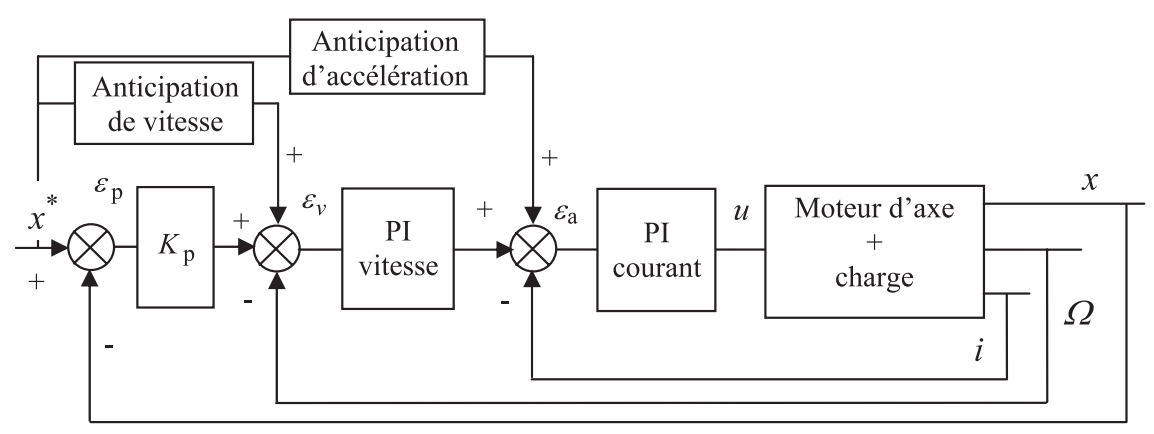

Fig. 3. Structure classique de commande d'axe.

Tableau 1. Récapitulatif des paramètres nécessaires à la modélisation pour $R=0,42 \Omega, L=5,4 \mathrm{mH}, K_{\mathrm{c}}=2,48 \mathrm{Nm} . \mathrm{A}^{-1}$, $K_{\mathrm{e}}=0,86 \mathrm{rad} \cdot \mathrm{s}^{-1} \cdot \mathrm{V}^{-1}, T_{\mathrm{e} i}=0,03 \mathrm{~ms}, T_{\mathrm{e} v}=T_{\mathrm{e} \mathrm{p}}=6 \mathrm{~ms}$.

\begin{tabular}{cccc}
\hline Axe & $X$ & $Y$ & $Z$ \\
\hline$f\left(\mathrm{Nm} \cdot \mathrm{rad}^{-1} \cdot \mathrm{s}\right)$ & 0,051 & 0,035 & 0,039 \\
$J_{\text {tot }}\left(\mathrm{kg} \cdot \mathrm{m}^{2}\right)$ & 0,032 & 0,024 & 0,027 \\
$K_{i \mathrm{c}}\left(\mathrm{V} \cdot \mathrm{A}^{-1}\right)$ & 13 & 12 & 12 \\
$T_{i \mathrm{c}}(\mathrm{ms})$ & 2 & 2 & 2 \\
\hline
\end{tabular}

\begin{tabular}{cccc}
\hline Axe & $X$ & $Y$ & $Z$ \\
\hline$K_{i v}\left(\mathrm{Nm} \cdot \mathrm{rad}^{-1} . \mathrm{s}\right)$ & 5 & 4,5 & 5,5 \\
$T_{i v}(\mathrm{~ms})$ & 4 & 6 & 4 \\
$K_{\mathrm{p}}\left(\mathrm{m} \cdot \mathrm{min}^{-1} \cdot \mathrm{mm}^{-1}\right)$ & 1,5 & 1,5 & 3,5
\end{tabular}

Pour la transmission :

$$
H_{\Omega x}(s)=\frac{\mu}{s}
$$

avec : $R, L$ résistance et inductance de l'induit, $K_{\mathrm{c}}, K_{\mathrm{e}}$ constante de couple et constante de force contreélectromotrice, $\mu$ constante incluant le rapport de réduction et le pas de la vis à bille, $u, i$ tension et courant d'induit, $\Omega, x$ vitesse angulaire de l'arbre moteur et position linéaire de l'axe, $f$ coefficient de frottement visqueux. $J_{\text {tot }}$ est l'inertie globale ramenée sur l'arbre moteur :

$J_{\text {tot }}=J_{\text {mot }}+J_{\text {réd }}+\frac{J_{\text {vis }}}{\eta^{2}}+\frac{J_{\text {axe }}}{\eta^{2}}$

$$
\text { ( } \eta \text { rapport de réduction) }
$$

avec $J_{\text {mot }}, J_{\text {réd }}, J_{\text {axe }}, J_{\text {vis }}$ les inerties respectives du moteur, du réducteur, de l'axe entraîné et de la vis à bille.

Les boucles de commande viennent ensuite se greffer sur cette modélisation de la structure d'un axe, le tout conduisant au schéma de la figure 3. Les variateurs sur le marché incluent généralement une structure cascade courant/vitesse/position et des termes d'anticipation en vitesse et accélération, le tout implanté numériquement. Les régulateurs des boucles de courant et vitesse sont classiquement de type PI(D), le régulateur de la boucle de position n'est souvent qu'un simple gain, nécessitant ces termes d'anticipations pour compenser les erreurs de traînage. Des modules supplémentaires de filtrage ainsi que des non-linéarités (saturation par exemple) viennent compléter cette modélisation, ils ne sont pas reproduits ici pour alléger la représentation.

De nombreux paramètres nécessaires à l'élaboration de l'environnement virtuel sont donnés dans les documents constructeurs ou renseignés au niveau de la commande numérique. Le tableau 1 récapitule les paramètres

considérés lors de la modélisation du centre d'usinage MIKRON.

\subsection{Identification}

Malgré tout, certaines caractéristiques comme les coefficients de frottement ou encore les inerties totales de chacun des axes demeurent inconnues. Pour les déterminer, une procédure d'identification en boucle fermée par moindres carrés a été développée [4], la structure du centre d'usinage ne permettant pas la réalisation d'essais en boucle ouverte. Les essais réalisés considèrent alors une configuration la plus simple possible, la machine étant à vide, avec des structures de commande sans anticipation, pour des déplacements linéaires sur un seul axe à la fois et exploitant les mesures disponibles de courant, vitesse, position.

Le modèle discret de la partie mécanique Éq. (2) est obtenu simplement par transformée d'Euler sous la forme :

$$
\begin{array}{r}
\frac{\Omega(z)}{I(z)}=\frac{K_{\mathrm{c}} / f}{\left(1+\frac{J_{\mathrm{tot}}}{f T_{\mathrm{e}}}\right)-\frac{J}{f T_{\mathrm{e}}} z^{-1}}=\frac{a}{1-b z^{-1}} \\
\left(z^{-1} \text { opérateur retard }\right)
\end{array}
$$

où $T_{\mathrm{e}}$ est la période d'échantillonnage. Sur un horizon de $n$ mesures, l'ensemble se traduit sous la forme matricielle suivante :

$$
A X=B
$$




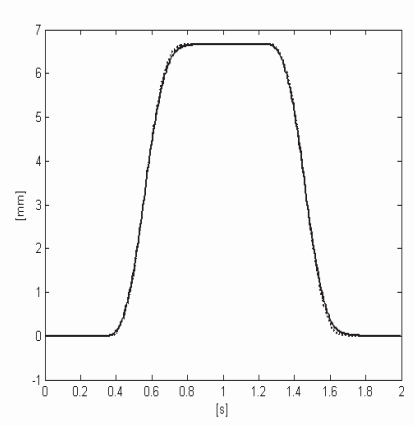

a)

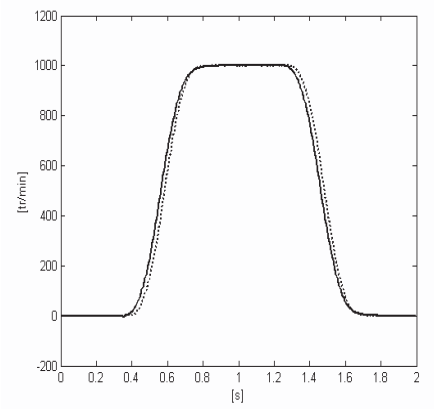

b)

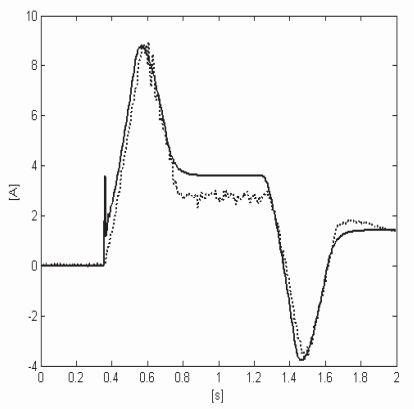

c)

Fig. 4. Déplacement linéaire sur l'axe $X$. Trait continu : simulation, trait pointillé : mesure sur la machine-outil. a) Erreur de poursuite. b) Vitesse. c) Courant.

avec $\boldsymbol{A}, \boldsymbol{B}, \boldsymbol{X}$ respectivement le régresseur, le vecteur des mesures et le vecteur des paramètres à estimer :

$$
\begin{aligned}
\boldsymbol{X}=\left[\begin{array}{l}
a \\
b
\end{array}\right]^{\prime} ; \boldsymbol{B}=\left[\begin{array}{c}
\Omega(1) \\
\vdots \\
\Omega(n)
\end{array}\right]^{\prime} ; \\
\boldsymbol{A}=\left(\begin{array}{cc}
i(1) & \Omega(0) \\
\vdots & \vdots \\
i(k) & \Omega(k-1) \\
\vdots & \vdots \\
i(n) & \Omega(n-1)
\end{array}\right), k=1, \cdots, n
\end{aligned}
$$

L'estimation de $\boldsymbol{X}$ minimisant la norme de l'erreur $\|\boldsymbol{A} \boldsymbol{X}-\boldsymbol{B}\|^{2}$ pour les $n$ points de mesure fournit la solution sous la forme $\boldsymbol{X}=\left[\boldsymbol{A}^{\prime} \boldsymbol{A}\right]^{-1} \boldsymbol{A}^{\prime} \boldsymbol{B}$, ce qui achève la détermination des paramètres inconnus $f$ et $J_{\text {tot }}$. Les valeurs numériques des paramètres identifiés sont données tableau 1.

\subsection{Validation}

La validation de cet environnement virtuel a été réalisée par comparaison du comportement obtenu en simulation avec celui relevé lors d'essais effectués sur la machine. Pour cela, plusieurs trajectoires types ont été testées, tout d'abord des trajectoires linéaires axe par axe, ensuite des trajectoires circulaires dans les différents plans, enfin des trajectoires tridimensionnelles quelconques. La même consigne que celle demandée à la machine-outil est appliquée en simulation, les structures de commande ainsi que les paramètres sont identiques à ceux décrits précédemment. Dans tous les cas, le comportement obtenu en simulation s'avère très proche de celui constaté sur la machine, tout particulièrement concernant la vitesse et la position. Seule l'allure du courant simulé s'écarte légèrement du courant mesuré, principalement à cause de l'hypothèse de modélisation par un moteur à courant continu, ce qui s'avère par ailleurs peu gênant pour l'analyse du comportement en positionnement. À titre d'exemple, la figure 4 suivante illustre cette comparaison dans le cadre d'un déplacement sur l'axe $X$, sur une distance de $15 \mathrm{~cm}$ à une vitesse linéaire de $10 \mathrm{~m} \cdot \mathrm{min}^{-1}$.

\section{Implantation de lois de Commande Prédictive Généralisée (GPC)}

L'outil virtuel précédemment développé permet désormais de tester de façon réaliste l'impact de nouvelles structures de commande. Celles-ci doivent être capables d'assurer un suivi de consigne à vitesses élevées (UGV par exemple) sans erreur de poursuite. Notons que minimiser les erreurs de poursuite sur chaque axe implique la minimisation de l'erreur de contour, définie comme la déviation orthogonale par rapport à la trajectoire souhaitée [5]. Par ailleurs, la démarche globale s'inscrivant dans un contexte d'architecture ouverte, il est nécessaire que toute structure de commande implantée dans la CN puisse se représenter par un formalisme unique. Il s'avère dès lors que la représentation générique dite « RST $»$ de toute loi de commande numérique répond au problème, puisqu'il est possible sous cette forme unique de représenter aussi bien les régulateurs PI classiques que les correcteurs prédictifs.

\subsection{Développements théoriques}

Ce paragraphe a pour but de rappeler les principes de la structure RST et de la Commande GPC. Le lecteur pourra consulter [6] pour plus de détails sur les avantages de cette structure RST, et [7] pour la mise en équation de l'algorithme prédictif.

\subsubsection{Le formalisme « RST »}

Ce formalisme générique fondamental permet donc de représenter toute loi de commande numérique par trois polynômes $R, S$ et $T$, sous la forme indiquée figure 5 . 


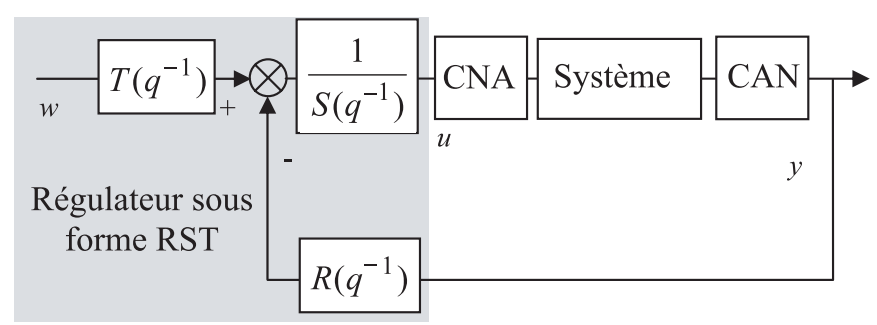

Fig. 5. Structure de commande numérique sous forme «ST».

Cette structure est dite à deux degrés de liberté, le polynôme $T$ (lorsqu'il est différent du polynôme $R$ ) permettant de dissocier la dynamique de suivi de trajectoire de la dynamique de rejet de perturbation.

La loi de commande est alors implantée dans la CN par l'équation aux différences :

$$
S\left(q^{-1}\right) u(t)=T\left(q^{-1}\right) w(t)-R\left(q^{-1}\right) y(t)
$$

Ainsi, un régulateur PI classique (de gain proportionnel $K$ et de constante intégrale $T_{i}$ ) peut se formaliser selon la structure de la figure 5 avec les polynômes suivants [6] :

$$
\begin{aligned}
& S\left(q^{-1}\right)=1-q^{-1} ; \\
& \quad R\left(q^{-1}\right)=T\left(q^{-1}\right)=K\left[\left(1+\frac{T_{\mathrm{e}}}{T_{i}}\right)-q^{-1}\right]
\end{aligned}
$$

\subsubsection{Structure et implantation de la Commande Prédictive Généralisée}

La philosophie de la Commande Prédictive Généralisée se fonde sur quatre grandes idées reproduisant les mécanismes décisionnels de base du comportement humain [8] : création d'un effet anticipatif par exploitation de la trajectoire à suivre dans le futur, définition d'un modèle numérique de prédiction, minimisation d'un critère quadratique à horizon fini, principe de l'horizon fuyant. On considère alors les points suivants [9] :

- le modèle du processus peut prendre différentes représentations (par fonction de transfert, par variables d'état), la structure adoptée ici est la forme CARIMA :

$$
A\left(q^{-1}\right) y(t)=B\left(q^{-1}\right) u(t-1)+\xi(t) / \Delta\left(q^{-1}\right)
$$

où $\xi(t)$ est un bruit blanc centré, $\Delta\left(q^{-1}\right)=1-q^{-1}$ garantissant la présence d'une action intégrale dans le régulateur, $A$ et $B$ polynômes définis à l'aide de l'opérateur retard $q^{-1}$ :

$$
\begin{aligned}
& A\left(q^{-1}\right)=1+a_{1} q^{-1}+\ldots+a_{n_{a}} q^{-n_{a}} \\
& \quad B\left(q^{-1}\right)=b_{0}+b_{1} q^{-1}+\ldots+b_{n_{b}} q^{-n_{b}}
\end{aligned}
$$

- le prédicteur optimal basé sur le modèle du processus est défini de façon unique par résolution d'équations diophantiennes :

$$
\begin{aligned}
\hat{y}(t+j)=\underbrace{F_{j}\left(q^{-1}\right) y(t)+H_{j}\left(q^{-1}\right) \Delta u(t-1)}_{\text {réponse libre }} \\
+\underbrace{G_{j}\left(q^{-1}\right) \Delta u(t+j-1)}_{\text {réponse forcée }}
\end{aligned}
$$

- l'élaboration de la commande optimale est obtenue par minimisation d'un critère quadratique, somme pondérée des erreurs de prédiction et des incréments de commande futurs :

$$
\begin{array}{r}
J=\sum_{j=N_{1}}^{N_{2}}[w(t+j)-\hat{y}(t+j)]^{2}+\lambda \sum_{j=1}^{N_{u}}[\Delta u(t+j-1)]^{2} \\
\text { avec } \Delta u(t+j)=0 \text { pour } j \geq N_{u}
\end{array}
$$

où $N_{1}, N_{2}$ sont l'horizon de prédiction inférieur et supérieur sur la sortie, $\lambda$ est le coefficient de pondération sur la commande, $N_{u}$ l'horizon de prédiction sur la commande.

Seule la première valeur de cette séquence est appliquée sur le système, la procédure étant itérée de nouveau à la période d'échantillonnage suivante selon le principe de l'horizon glissant. La structure ainsi obtenue se représente finalement sous la forme « RST » Éq. (8) adéquate à l'architecture ouverte. Les polynômes $R, S$ et $T$ sont élaborés hors ligne et définis de façon unique dès lors que le modèle et les quatre paramètres de réglage sont figés. Le temps de calcul est de plus très faible, correspondant au calcul de la commande via une équation aux différences de faible degré. Cet aspect permet l'implantation de cette stratégie dans le cas de faibles périodes d'échantillonnage, pour l'UGV par exemple. On note également que le polynôme $T$ possède une structure non causale, assurant ainsi un effet anticipatif en boucle fermée. Les termes d'anticipation présents dans les CN classiques et complexes à mettre au point deviennent en conséquence inutiles.

Pour les besoins de la commande numérique représentée figure 3 , une version prédictive cascade vitesse-position est alors implantée (voir [7] pour le principe de la structure cascade prédictive), en conservant les boucles de courant de dynamique très rapide avec leur régulateur $\mathrm{PI}$.

\subsection{Résultats de simulation}

Le but est ici de comparer les performances obtenues sur la machine dans sa configuration classique de commande (cascade PI de courant, PI de vitesse, gain de position et anticipation de vitesse et d'accélération), pour une consigne interpolée linéairement et une vitesse évoluant selon un profil trapézoïdal, à celles mises en 


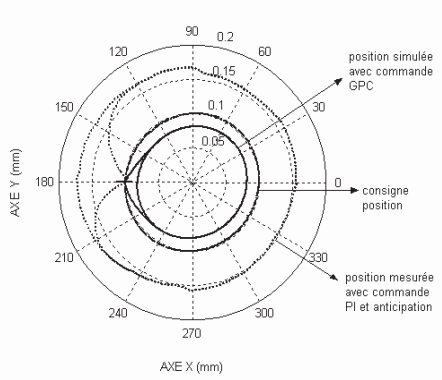

a)

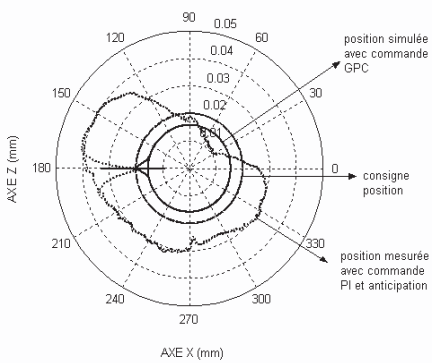

b)

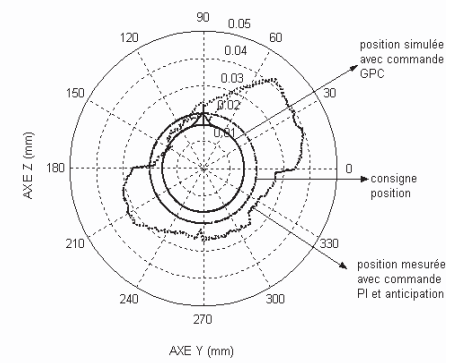

c)

Fig. 6. Consigne (trait continu), position mesurée « avec PI (trait pointillé), position simulée « avec GPC » (trait continu). a) Plan $X Y$, b) plan $X Z$, c) plan $Y Z$.

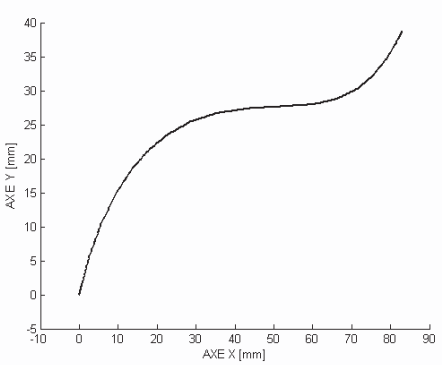

a)

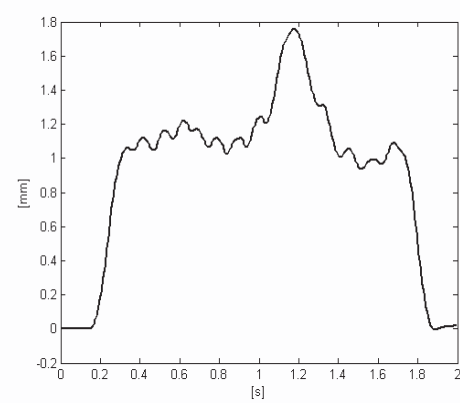

b)

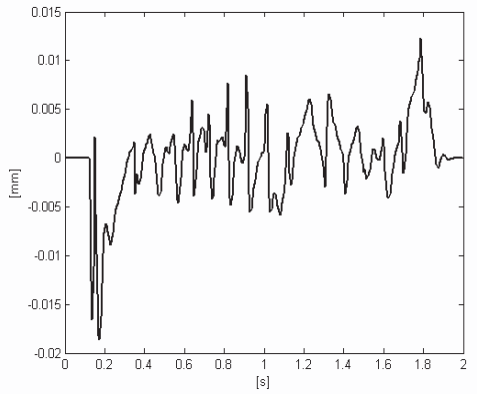

c)

Fig. 7. a) Consigne dans le plan $X Y$, b) erreur mesurée PI, c) erreur simulée GPC.

évidence dans l'environnement virtuel. Pour ce dernier, la commande est celle indiquée à la section précédente et la consigne exactement la même que celle générée par la machine au niveau de chacun des axes. Les paramètres de réglage des régulateurs GPC ont été choisis selon des conditions de robustesse et stabilité indiquées dans [7], soit $N_{1}=N_{u}=1, N_{2}=4$ identiques pour les trois axes et les deux boucles, $\lambda_{\mathrm{e}}=1 ; \lambda_{i}=0,28$ pour l'axe $X, \lambda_{\mathrm{e}}=$ 1,$76 ; \lambda_{i}=0,29$ pour l'axe $Y$ et $\lambda_{\mathrm{e}}=1,29 ; \lambda_{i}=0,27$ pour l'axe $Z$. Comme précédemment, plusieurs trajectoires types ont été testées, tout d'abord des trajectoires linéaires axe par axe, ensuite des trajectoires circulaires dans les différents plans, enfin des trajectoires tridimensionnelles quelconques.

La figure 6 montre les sorties en position dans le cas de consignes circulaires de rayon $100 \mathrm{~mm}$, pour une vitesse linéaire de déplacement de $10 \mathrm{~m} \cdot \mathrm{min}^{-1}$. Un zoom est effectué sur la partie extérieure de ces trajectoires afin de visualiser les erreurs résultantes. On constate dans les trois plans considérés que la commande prédictive améliore sensiblement les performances en suivi par rapport à la commande classique, l'erreur étant réduite de 60 à 20 microns dans le plan $X Y$, de 40 (resp. 20) à 5 microns pour le plan $Y Z$ (resp. $X Z$ ). Ces progrès sont significatifs, même s'ils demandent bien sûr à être corroborés par des essais sur la machine.

La figure 7 a montre la position en réponse à une trajectoire dans le plan $X Y$. Les figures $7 \mathrm{~b}$ et $7 \mathrm{c}$ illustrent les modules des erreurs spatiales obtenues pour la commande classique (amplitude d'erreur de 1,76 mm) et pour la commande GPC (amplitude d'erreur de 31 microns). Ces résultats permettent d'aboutir aux mêmes conclusions que ci-dessus.

Les essais précédents montrent qu'il est possible d'améliorer les performances grâce à la structure de commande, mais l'on constate alors (courbe 7c) les limitations (discontinuités liées à la génération) induites par la structure actuelle de la génération de trajectoire (même si une interpolation plus fine avait été sélectionnée). C'est pourquoi il apparaît également nécessaire d'inclure dans le cadre de l'architecture ouverte un module avancé de génération de consignes (Fig. 1).

\section{4 Évolution de l'architecture pour l'intégration d'un format surfacique}

Lors du processus de réalisation des pièces de forme complexe, c'est la trajectoire de l'outil qui est décrite et transmise au directeur de commande numérique et non pas la géométrie à obtenir. Actuellement, seuls les formats de description linéaires ou polynomiaux sont intégrés dans le directeur de commande numérique, ce qui entraîne une dégradation du modèle surfacique décrivant la géométrie de la surface en un ensemble de points. Dans le cas de l'interpolation linéaire, ces points sont des points de passage de l'outil au contact de la surface. La trajectoire de l'outil est linéaire par morceaux, présentant des discontinuités $C^{1}$ (Fig. 7c). Il en résulte alors des erreurs de corde entre deux points et des performances dynamiques médiocres 


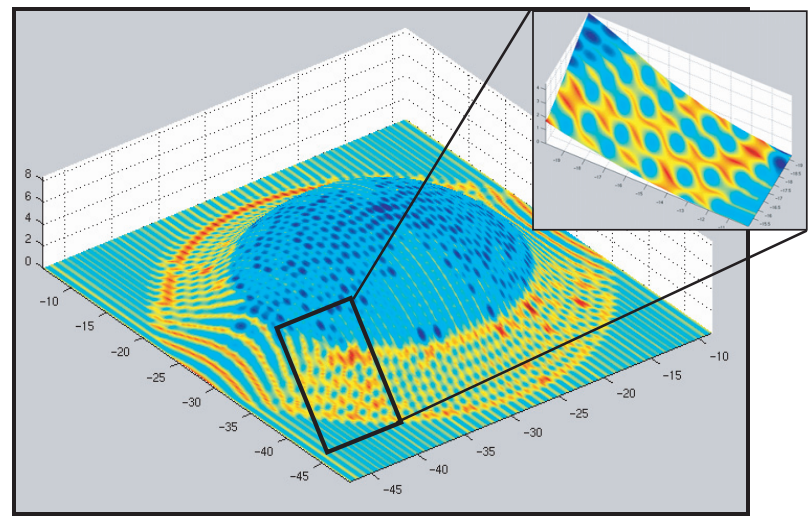

a)

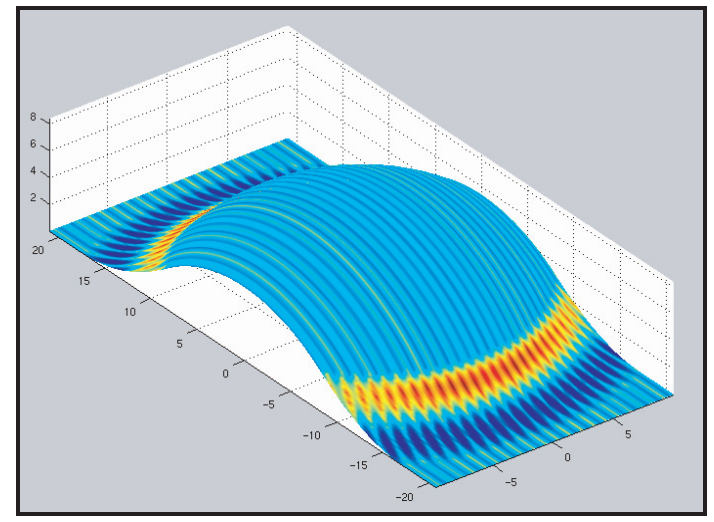

b)

Fig. 8. a) Peau d'orange en interpolation linéaire, b) résultats en format Nurbs (Siemens Poly 3).

au voisinage des discontinuités, ce qui est pénalisant dans le cadre de l'UGV [10]. Dans le cas de l'interpolation polynomiale, les points calculés sont interpolés par une courbe qui est modélisée par ses points de contrôle. Il en résulte également des erreurs de corde puisque la trajectoire n'est pas contrainte à rester sur la surface nominale du modèle CAO. Cependant, les performances dynamiques sont meilleures qu'en interpolation linéaire [11]. Quel que soit le format de description, l'ensemble de points est transmis au directeur de commande numérique qui réalise la préparation de la trajectoire et la commande en temps réel des axes de la machine outil [10].

Afin d'améliorer la qualité des surfaces usinées, nous envisageons de communiquer au directeur de commande numérique des trajectoires d'usinage sous forme surfacique. Les trajectoires d'usinage sont définies dans l'espace paramétrique 2D de cette surface et leur représentation tridimensionnelle appartient de fait à celleci. Les écarts dus aux approximations lors du calcul des trajectoires disparaissent. Il n'y a ni erreur de posage de l'outil sur la surface, ni erreur de corde et la maîtrise de la hauteur des crêtes laissées sur la pièce est plus facile [12]. C'est une extension de l'interpolation polynomiale de courbes 3D actuelle avec un paramètre supplémentaire.

Le projet STEP-NC, qui a pour but d'établir un nouveau format de description des trajectoires d'usinage entre le système de FAO et la commande numérique, est un support possible pour parvenir à notre objectif [13] Cependant il n'est à l'heure actuelle qu'à l'état de projet, et aucun constructeur ne propose de CN capable d'interpréter un format surfacique. Ce type d'interpolation a cependant été testé récemment en employant un simple ordinateur muni de cartes d'axes et relié à une structure à 3 axes [13]. L'objectif était de maintenir une vitesse d'avance constante au niveau du point de contact outil surface, mais il ne s'agissait pas d'une véritable machine d'usinage. Il ne nous a pas semblé raisonnable d'utiliser cette approche dans le cas d'une machine d'usinage à grande vitesse à cause des difficultés d'intégration des parties commandes et opératives. Les gains sur la qualité géométrique des trajectoires auraient été masqués par les imperfections de la commande. Une architecture ouverte nous permettrait donc d'implanter des nouveaux générateurs de trajectoire en FAO et de consignes dans la CN.

Afin de prouver l'intérêt d'une approche surfacique, nous avons donc exploité les formats de trajectoires disponibles dans les directeurs de commande numérique. Nous parvenons à éliminer l'effet de peau d'orange dû à la désynchronisation des passes et aux erreurs de corde en utilisant un format NURBS de description des trajectoires d'usinage disponible sur la commande numérique SIEMENS 840D (fonction POLY) [14]. La validation virtuelle des avantages du format par rapport à l'interpolation linéaire est montrée grâce à la simulation d'usinage au moyen du $Z$-buffer (Fig. 8a). Seules subsistent des erreurs de corde au voisinage des raccordements car le format d'interpolation POLY degré 3 ne permet pas d'être parfaitement posé sur la surface (Fig. 8b). Un polynôme de degré 5 aurait permis d'annuler cette erreur.

\section{Conclusions}

La mise en œuvre d'une stratégie de commande avancée de type prédictif pour la commande d'axes de machines-outils apporte une amélioration incontestable des performances en positionnement et, par son anticipation en boucle fermée, évite un réglage complexe et sensible de compensations en boucle ouverte. La simplicité d'implantation et le faible temps de calcul résultant en ligne en font une structure adaptée aux applications pour lesquelles des spécifications sévères sont imposées, comme pour l'UGV. Par ailleurs, cette loi de commande s'inscrit dans le contexte général des lois numériques polynomiales « RST », de sorte que dans le cadre d'une architecture ouverte, un seul module de commande générique peut s'envisager. Malgré tout, et à cause du caractère fermé des CN actuelles, cette amélioration des performances n'a pu être illustrée qu'à partir d'un environnement virtuel, obtenu par modélisation et identification du comportement réel de la machine-outil servant de support à l'étude. Même si 
les résultats s'avèrent pertinents, une ouverture de la $\mathrm{CN}$ est souhaitable afin d'effectuer les essais dans un environnement réel.

\section{Références}

[1] G. Pritschow, Open Controller Architecture - Past, Present and Future, Annals of the CIRP 50/2 (2001) 463-470

[2] Y. Koren, Z.J. Pasek, A. Galip Ulsoy, U. Benchetrit, Real-Time Open Control Architectures and System Performance, Annals of the CIRP 45/1 (1996) 377-380

[3] M. Susanu, D. Dumur, C. Lartigue, Modelling and advanced control of machine- tool in an open architecture framework, 14th International conference on Control Systems and Computer Science, Bucarest, 2003

[4] K. Erkorkmaz, Y. Altintas, High speed system design. Part II : modelling and identification of feed drives, Int. J. Machine Tools \& Manufacture 41 (2001) 1487-1509

[5] K. Erkorkmaz, Y. Altintas, High speed system design. Part III : high speed tracking and contouring control of feed drives, Int. J. Machine Tools \& Manufacture 41 (2001) 1637-1658

[6] D. Dumur, P. Boucher, Coordination par la position et la vitesse, commande de moteurs des machines-outils, Chapitre $11 \mathrm{du}$ volume Méthodologie de la commande des machines électriques du traité IGEM-Électronique,
Génie Électrique et Microélectronique, Coordinateur René Husson, Hermès, 2003

[7] P. Boucher, D. Dumur, La commande prédictive, Éditions Technip, Paris, 1996

[8] J.A. Rossiter, Model - based predictive control, A practical approach, CRC Press, ISBN 0-8493-1291-4, 2003

[9] D.W. Clarke, C. Mohtadi, P.S. Tuffs, Generalised Predictive Control : Part I The Basic Algorithm, Part II : Extensions and Interpretation, Automatica 23-2 (1997) $137-160$

[10] A. Dugas, CFAO et Usinage - Simulation d'usinage de formes complexes, Doctorat de l'École centrale de Nantes et de l'Université de Nantes, décembre 2002

[11] E. Duc, C. Lartigue, S. Laporte, Assessment of the description format of tool trajectories in 3-axis HSM of sculptured surfaces, Metal cutting and High speed machining, Kluwer Academic Publishers, 2002, pp. 363-375

[12] C. Tournier, Contribution à la conception des formes complexes : la surface d'usinage en fraisage à 5 axes iso crêtes, Doctorat École normale supérieure de Cachan, décembre 2001

[13] M.-C. Tsai, C.-W. Cheng, M.-Y. Cheng, A real-time NURBS surface interpolator for precision three-axis CNC machining, Int. J. Machine Tools \& Manufacture 43 (2003) 1217-1227

[14] M. Ritou, Intégration d'un modèle surfacique de trajectoires d'usinage dans la Commande Numérique, Mémoire de DEA de Production Automatisée, LURPA-ENS de Cachan, 2003 